\title{
Adults with congenital heart disease: ready for mobile health?
}

\author{
R. W. Treskes - M. Koole - D. Kauw - M. M. Winter - M. Monteiro · D. Dohmen - A. Abu-Hanna · M. P. Schijven · \\ B. J. Mulder · B. J. Bouma $\cdot$ M. J. Schuuring
}

Published online: 13 February 2019

(C) The Author(s) 2019

\begin{abstract}
Purpose Mobile health (mHealth) could improve the outcome of grown-up patients with congenital heart disease (GUCH) and reduce their emergency care utilisation. Inappropriate use of mHealth, however, can lead to data overload for professionals and unnecessary data collection for patients, increasing the burden for both. We aimed to determine the clinical characteristics of patients with high emergency care utilisation and to test whether these patients were willing to start using mHealth.

Methods Clinical characteristics and emergency care utilisation of consecutive GUCH patients who visited one of the two participating cardiologists at the out-
\end{abstract}

\section{R. W. Treskes}

Department of Cardiology, Leiden University Medical Centre, Leiden, The Netherlands

M. Koole · D. Kauw · M. M. Winter · M. Monteiro · B. J. Mulder · B. J. Bouma · M. J. Schuuring ( $ه)$

Department of Cardiology, Academic Medical Centre, Amsterdam, The Netherlands

m.j.schuuring@amc.uva.nl

M. Koole

Department of Cardiology, Rode Kruis Ziekenhuis, Beverwijk, The Netherlands

D. Dohmen

FocusCura, Driebergen-Rijsenburg, The Netherlands

\section{A. Abu-Hanna}

Department of Medical Informatics, Academic Medical

Centre, Amsterdam, The Netherlands

\section{P. Schijven}

Department of Surgery, Academic Medical Centre,

Amsterdam, The Netherlands

\section{J. Schuuring}

Department of Cardiology, Haga Teaching Hospital, The

Hague, The Netherlands patient clinic of the Academic Medical Centre in Amsterdam were studied retrospectively. All patients were approached to fill in an mHealth questionnaire. A frequency of three or more emergency visits in 5 years was defined as high emergency care utilisation.

Results In total, 202 consecutive GUCH patients who visited one of the two participating cardiologists were studied. Median age was 41 years, $47 \%$ were male, and $51 \%$ were symptomatic. In the previous 5 years, 134 emergency visits were identified. Of all patients, $8 \%$ had high emergency care utilisation. High emergency care utilisation was associated with patients being symptomatic, using antiarrhythmic drugs or diuretics. In total, $75 \%$ of all patients with high emergency care utilisation were willing to start using mHealth.

Conclusion GUCH patients who are symptomatic, those on antiarrhythmic drug therapy and those on diuretics are suitable candidates for enrolment in future mHealth initiatives because of both high care utilisation and high motivation to start using mHealth.

Keywords Congenital heart disease · GUCH · Resource utilisation - Delivery of healthcare - Mobile health . eHealth

\section{What's new}

- Telemonitoring with mobile phones is promising, but research remains to be done.

- Grown-up patients with congenital heart disease have a proven interest in mobile health.

- This study identifies the characteristics of patients with high healthcare use.

- The vast majority of these patients is in possession of a smartphone and willing to start using mobile health. 


\section{Introduction}

Congenital heart disease (CHD) is one of the most common birth defects [1-3]. During the past decades, the life expectancy of children born with a CHD has increased dramatically. At present, 95\% of children with CHD reach adulthood [1]. However, many of the grown-ups with congenital heart disease (GUCH) are chronically affected by residual sequelae leading to unpredictable arrhythmias, heart failure and a reduced quality of life [4-8]. In general, GUCH patients have a high utilisation of emergency resources, with emergency care utilisation increasing as age progresses [4]. As the population of GUCH patients is increasing in number and age, total emergency care utilisation of this population is expected to increase [9].

Mobile health (mHealth) is the provision of medical care by mobile technologies capable of delivering health information, monitoring clinical signs and enabling direct care and patient education [10]. Using mobile technology, vital signs can be collected and sent immediately to a treating cardiologist. E-visits enable immediate and remote contact between doctor and patient [11]. Therefore, potential benefits of mHealth include: rapid delivery of round-the-clock care; enhanced daily monitoring and hence timely response and more convenience for patients; and improved access for patients [12]. In order to improve outcome and reduce emergency care utilisation, careful selection of patients that are most likely to benefit from an mHealth intervention is warranted. If used in an inappropriate patient population, mHealth can lead to data overload for healthcare professionals and unnecessary data collection for patients, increasing the burden for both [13]. Patients with a high emergency care utilisation and high motivation to start using mHealth are suitable candidates to include in new mHealth initiatives. It is therefore the primary objective of this study to determine the clinical characteristics of GUCH patients with high emergency care utilisation. It is the secondary objective to combine these findings with the results of an mHealth questionnaire, to test whether GUCH patients with high emergency care utilisation are willing to start using mHealth.

\section{Methods}

\section{Population and data collection}

For this study, two cardiologists specialised in GUCH (B.B. and B.M.) approached consecutive patients who had an appointment at the outpatient clinic with them to fill in an mHealth questionnaire. These patients visited the outpatient clinic at the Academic Medical Centre in Amsterdam between April 2016 and September 2016. Clinical characteristics and emergency care utilisation of these GUCH patients were studied retrospectively. Clinical characteristics noted were: severity of the CHD (in accordance with the Bethesda conference) [14], history of cardiac surgery, history of pacemaker or implantable cardioverter defibrillator (ICD) implantation and the use of diuretics or any antiarrhythmic drug therapy. In patients receiving antiarrhythmic drug therapy, the indication was noted as well. Beta-blockers were considered an antiarrhythmic drug therapy if the drug was initiated or the dose was altered for symptoms of palpitations or treatment for arrhythmia control. Cardiac-related symptoms were rated in accordance with the New York Heart Association (NYHA) Functional Classification. GUCH patients with a NYHA class II or higher were considered symptomatic. Emergency care utilisation was defined as visits to the emergency room, cardiac care unit or unplanned outpatient clinic visits. Outpatient clinic visits were counted if they included a visit to a cardiologist, cardiologist in training, heart failure nurse or dedicated CHD nurse at the Department of Cardiology of the Academic Medical Centre. An outpatient clinic visit was considered 'unplanned' if the electronic medical record explicitly stated that the patient was seen without a scheduled appointment in case of symptoms. Interventions noted following an emergency care visit were any type of openheart surgery, aneurysm surgery, pacemaker or ICD implantation or replacement, diagnostic catheterisations, electrical cardioversions (ECV), catheter-based interventions and bronchoscopy in case of haemoptysis. High care utilisation was defined as a score of three or more emergency visits between 1 June 2011 and 31 December 2016.

All patients were approached to fill in an mHealth questionnaire on paper. Details of the questionnaire have been described previously [15] Exclusion criteria were being mentally impaired (at physician's discretion), having no knowledge of the Dutch language or being younger than 18 years of age.

\section{Data management and statistics}

SPSS 22 (IBM Corp. Released 2013. IBM SPSS Statistics for Windows, Version 22.0. IBM Corp., Armonk, NY, USA) was used for statistical analysis. To identify GUCH patients who would most likely benefit from mHealth, determinants were set off against an emergency care utilisation of three or more emergency visits and/or interventions in the previous 5 years. Variables were compared with a chi-squared test. A $p$ value $\leq 0.05$ was considered statistically significant.

\section{Results}

\section{Population characteristics}

In total, 202 consecutive patients who visited the outpatient clinic and had an appointment with one of the two participating cardiologists (B.B. and B.M.) at the Academic Medical Centre in Amsterdam between 
Table 1 Comparison of high and low care utilisation. PM Pacemaker, ICD implantable cardioverter-defibrillator

\begin{tabular}{|c|c|c|c|c|}
\hline & All patients $(n=202)$ & $\begin{array}{l}\text { Low care utilisation }(n=186 \\
(92 \%))\end{array}$ & $\begin{array}{l}\text { High care utilisation }(n=16 \\
(8 \%))\end{array}$ & $p$ \\
\hline Median age, years & $41(18-78)$ & $40(18-78)$ & $42(23-77)$ & \\
\hline Male, $\%$ & $94(47)$ & $87(46)$ & $7(43)$ & 0.816 \\
\hline \multicolumn{5}{|l|}{ Congenital heart disease } \\
\hline Mild, \% & $39(19)$ & $35(19)$ & $4(25)$ & 0.548 \\
\hline Moderate, \% & $123(61)$ & $116(62)$ & $8(50)$ & 0.352 \\
\hline Severe, $\%$ & $40(20)$ & $35(19)$ & $4(25)$ & 0.548 \\
\hline \multicolumn{5}{|c|}{ New York Heart Association class } \\
\hline Class I, \% & $97(48)$ & $95(51)$ & $2(13)$ & $<0.001$ \\
\hline Class $\geq I I, \%$ & $105(52)$ & $91(49)$ & $14(87)$ & $<0.001$ \\
\hline \multicolumn{5}{|l|}{ Event history } \\
\hline Cardiac surgery, $\%$ & $168(83)$ & $156(83)$ & $12(75)$ & 0.363 \\
\hline PM/ICD implantation, \% & $17(8)$ & $14(8)$ & $3(19)$ & 0.121 \\
\hline \multicolumn{5}{|l|}{ Medication } \\
\hline Diuretics, \% & $19(9)$ & $12(6)$ & $7(44)$ & $<0.001$ \\
\hline Antiarrhythmic, \% & $62(31)$ & $51(27)$ & $11(69)$ & 0.001 \\
\hline \multicolumn{5}{|l|}{ mHealth } \\
\hline Smartphone utilisation (\%) & $186(92)$ & $172(92)$ & $14(87)$ & 0.369 \\
\hline Wiling to use mHealth (\%) & $143(71)$ & $131(70)$ & $12(75)$ & 0.70 \\
\hline
\end{tabular}

April 2016 and September 2016 were studied. Median age was 41 years (interquartile range $32-50$, range 18-78 years), $47 \%$ were male and $51 \%$ were symptomatic. Of all patients, $19 \%$ had mild CHD, $61 \%$ moderate CHD and $20 \%$ severe CHD. A total of $83 \%$ had a history of cardiac surgery and $8 \%$ had had a pacemaker or ICD implanted. Thirty-one per cent received

Table 2 Information on emergency visits

\begin{tabular}{|l|l|}
\hline Symptoms & $\boldsymbol{n}(\%)$ \\
\hline Palpitations & $55(41 \%)$ \\
\hline Chest pain & $32(24 \%)$ \\
\hline Fever & $16(12 \%)$ \\
\hline Fatigue & $13(10 \%)$ \\
\hline Shortness of breath & $7(5 \%)$ \\
\hline Haemoptysis & $6(4 \%)$ \\
\hline Neurological symptoms & $5(4 \%)$ \\
\hline Diagnoses & \\
\hline No diagnosis of cardiac nature & $62(46 \%)$ \\
\hline Arrhythmia & $50(37 \%)$ \\
\hline Endocarditis & $6(5 \%)$ \\
\hline Pulmonary hypertension & $6(5 \%)$ \\
\hline Stroke & $5(4 \%)$ \\
\hline Valvular heart disease & $3(2 \%)$ \\
\hline Heart failure & $2(1 \%)$ \\
\hline Therapeutic regimen consequences & \\
\hline No changes in therapeutic regimen & $59(44 \%)$ \\
\hline Medication changes & $52(39 \%)$ \\
\hline Electrocardioversion & $29(21 \%)$ \\
\hline Interventions & $4(3 \%)$ \\
\hline Planned interventions & $3(2 \%)$ \\
\hline
\end{tabular}

antiarrhythmic drug therapy and 9\% used diuretics (Tab. 1). Only $5 \%$ were in NYHA class IV. All patients filled in the mHealth questionnaire.

\section{Emergency visits}

In the previous 5 years, 202 patients accounted for 134 emergency visits, 59 (29\%) of whom had one or more emergency visit. Sixteen $(8 \%)$ of the 202 patients had high care utilisation and 186 (92\%) low care utilisation. No significant differences in gender, history of cardiac surgery or severity of CHD were found between patients with high and low care utilisation. Significant differences were found in NYHA class ( $87 \%$ vs $49 \%$, $p<0.001$ ), use of diuretics ( $44 \%$ vs $7 \%, p<0.001)$ and antiarrhythmic drug therapy $(69 \%$ vs $27 \%, p=0.001)$ (Tab. 1).

Tab. 2 and Fig. 1 show all of the symptoms with which patients presented, the subsequent diagnoses made, and the treatment administered. Most patients presented with either palpitations $(41 \%)$ or chest pain (24\%). In $46 \%$ of all cases, no diagnosis of a cardiac nature was made. In $37 \%$, a patient was diagnosed with an arrhythmia (Fig. 1).

Emergency visits resulted in a variety of different actions. In $44 \%$ of all cases the therapeutic regimen was not changed. Drug therapy was changed in 52 $(39 \%)$ cases. In 8 (15\%) out of 55 cases of palpitations, the therapeutic regimen was not changed. Therapeutic regimen changes included 16 (29\%) cases of ECV, $13(24 \%)$ cases of adjusting antiarrhythmic drug therapy after ECV, 7 (13\%) cases of adjusting antiarrhythmic drug therapy only, 10 (18\%) cases of initiating antiarrhythmic drug therapy and $1(2 \%)$ case of ra- 
Fig. 1 Frequency of emergency care visits, reasons and subsequent diagnoses
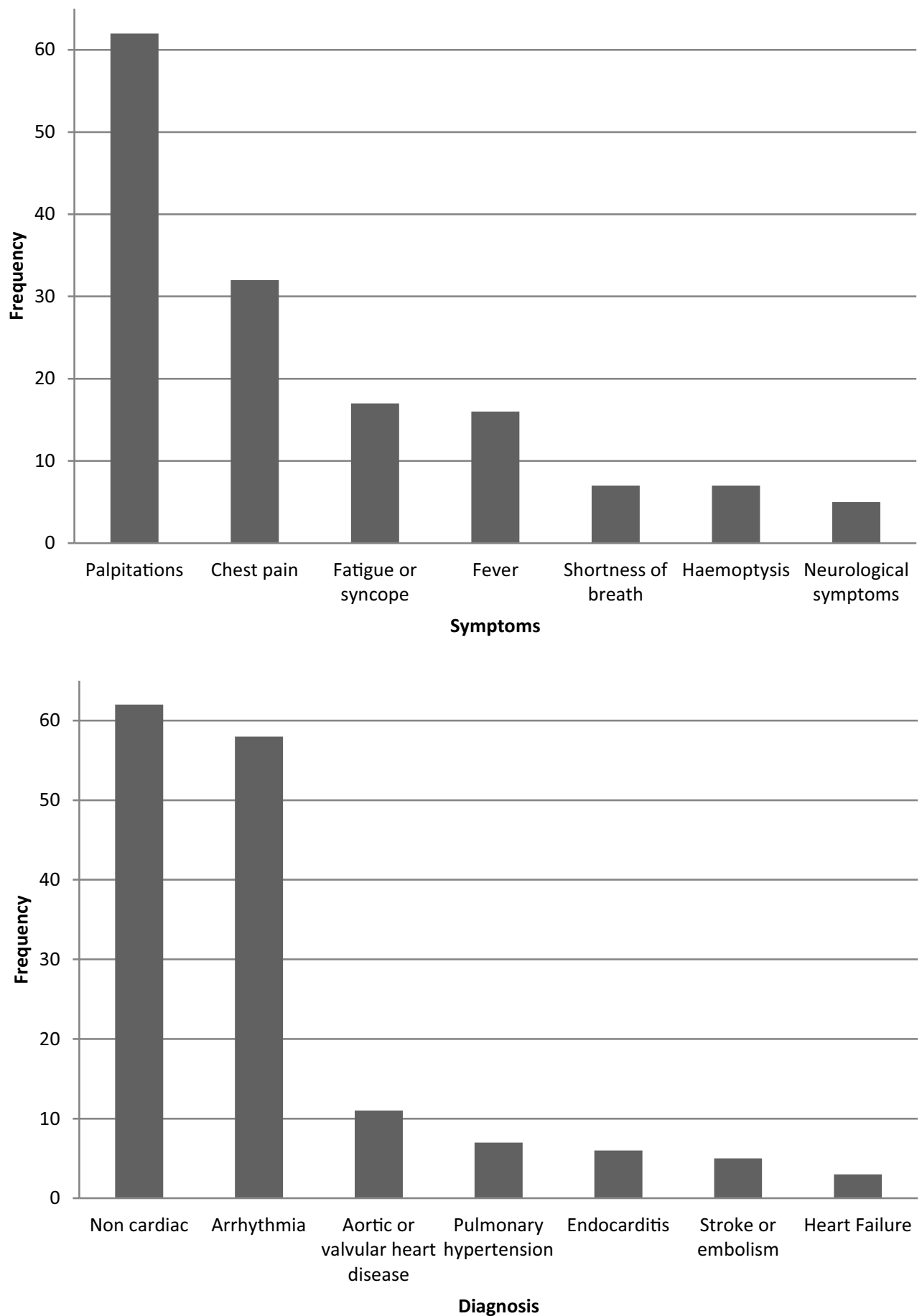

diofrequency ablation. In 29 (91\%) of the 32 cases of chest pain no action was taken. Therapeutic regimen changes included 3 (9\%) cases of initiating antibiotic treatment for the suspicion of endocarditis.

\section{Patient motivation to start using mHealth amongst patients with high emergency care utilisation}

In total, $16 \mathrm{GUCH}$ patients had high care utilisation. Median age was 46 years, $56 \%$ were female and $87 \%$ were symptomatic. Of all $202 \mathrm{GUCH}$ patients, $25 \%$ had a mild CHD, $50 \%$ a moderate and $25 \%$ a severe CHD. Antiarrhythmic drugs were used by $69 \%$ of patients and diuretics by $44 \%$.

Of all patients with high care utilisation, $87 \%$ were in possession of a smartphone and $18 \%$ claimed to use mHealth already. Of all patients, $44 \%$ wanted information about their disease, while $44 \%$ wanted lifestyle advice via mobile technology. A total of $56 \%$ were willing to fill in vital signs on their smartphone, $56 \%$ were willing to fill in symptoms on their smartphone, $62 \%$ wanted advice in case of aberrant vital 


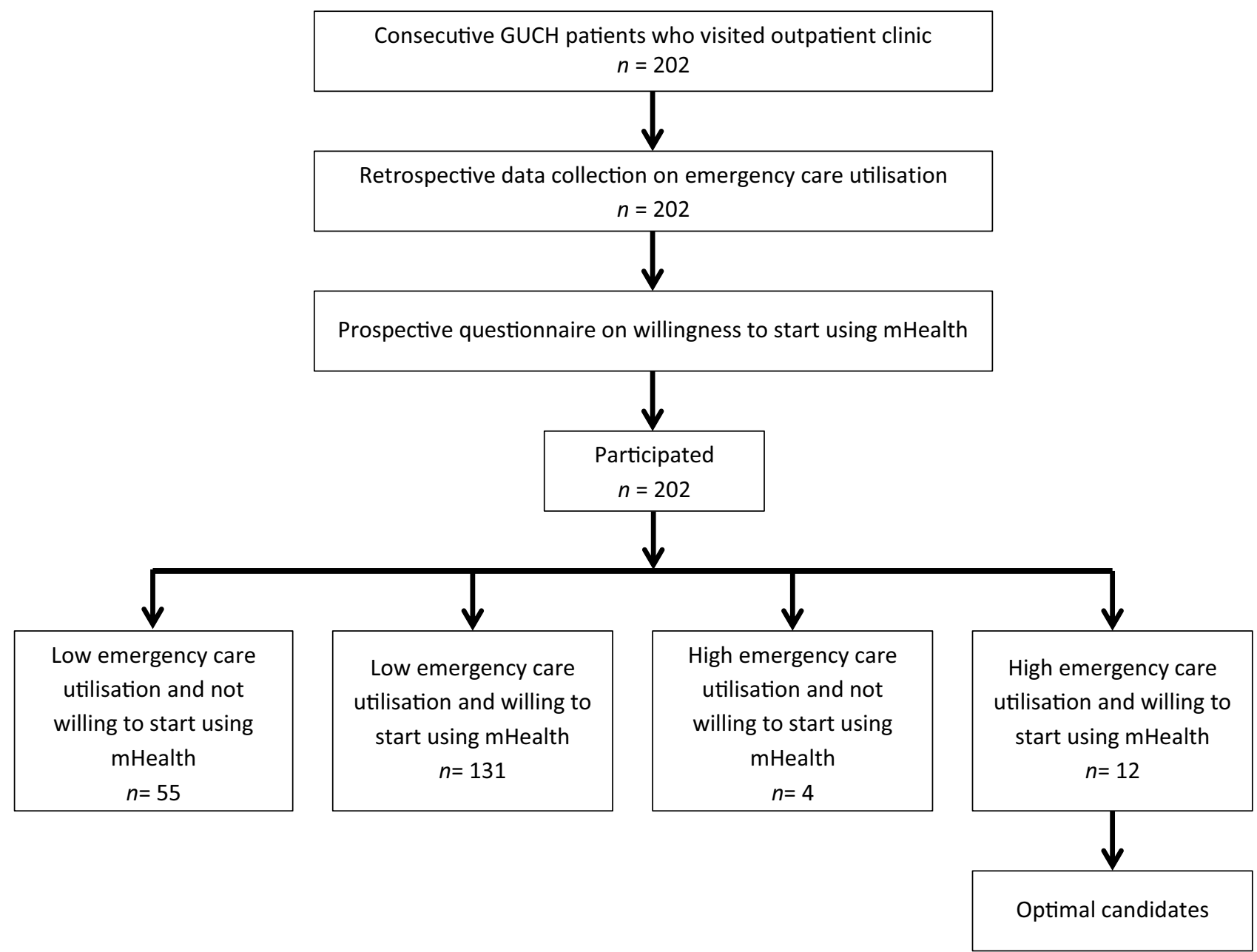

Fig. 2 Flow chart of patient selection. GUCH grown-up patients with congenital heart disease

signs, $62 \%$ wanted advice regarding symptoms of possible cardiac origin and $75 \%$ were willing to start using mHealth.

In contrast, in the low care utilisation group, 131 (70\%) patients were willing to start using mHealth (Fig. 2).

\section{Discussion}

To our knowledge this is the first study to determine the suitability of candidates for enrolment in new mHealth initiatives in GUCH patients. In our study, we found that symptomatic patients who are on diuretics or antiarrhythmic drug therapy are more likely to visit the emergency room. These patients might benefit from mHealth, as emergency visits could be prevented via mHealth. In patients with few emergency visits, mHealth is less likely to be beneficial as it is a priori less likely to prevent an emergency visit. Therefore, our study could help to avoid initiation of mHealth with the goal of decreasing emergency care utilisation in an inappropriate patient population and could prevent unnecessary data collection for patients. Furthermore, the therapeutic regimen was not changed at $44 \%$ of all emergency visits. The number of such visits might also be reducible via mHealth.

\section{Emergency care utilisation}

In this study, $29 \%$ of all participating GUCH patients had had an emergency visit in the previous 5 years. This percentage was lower than in the study of Mackie et al. [16] and that of Verheugt et al. [17], who reported that $68 \%$ and $50 \%$ of their study population had had an emergency visit, respectively. Definitions of emergency care utilisation between Mackie et al., Verheugt et al. and our study were comparable. It is therefore hypothesised that this difference is due to the fact that for our study, only emergency visits at the Academic Medical Centre were analysed. The Academic Medical Centre is a tertiary hospital, treating patients from a large geographic region. In emergency cases, these patients are more likely to visit a local hospital close to their homes. These emergency visits are not counted in this study. Therefore, the frequency of emergency visits could be higher in our study population. In our study most patients presented with palpitations and chest pain. Arrhythmias were the 
most common final diagnosis. Heart failure was diagnosed in only $1 \%$ of patients, which was lower than in the studies of Cedars et al. [18] and Negishi et al. [19]. There are several explanations for this difference. First, patients might have been admitted to other hospitals. Second, in our study, diagnoses were classified according to primary diagnosis. Some patients with arrhythmias presented with heart failure symptoms but were diagnosed in the 'arrhythmia category'. Third, two nurse practitioners specialised in heart failure had optimised treatment at the outpatient clinic, which could potentially have led to a reduction of deteriorations in heart function. Finally, in our study population, $31 \%$ had been hospitalised in the previous 5 years. This was in line with the study of Mackie et al. [16] and that of Moons et al. [20].

\section{Selecting GUCH patients for mobile health}

Our study showed that the majority of patients were willing to use mHealth applications. Several validated technologies that allow for remote electrocardiogram (ECG) monitoring and automatic transmission are already available [21] and easy to use. For the selection of the best candidates for possible future mHealth initiatives inclusion criteria should be: GUCH patients, experiencing frequent palpitations and/or chest pain, able to operate a smartphone and having high care utilisation. Furthermore, having severe CHD, using diuretics and/or antiarrhythmic drugs, having an implant or experiencing symptoms can be taken into account in selecting GUCH patients. Gender and age should not be a discriminant factor. Issues regarding privacy will need to be addressed, since this new technology will be sensitive as regards breach of privacy. Lastly, mHealth literacy is an important predictor of success in mHealth intervention [22]. Therefore, acceptability should be taken into account when initiating mHealth initiatives in this group.

Currently, several devices that allow a user to record an ECG are already available. These devices can be used by patients themselves and do not necessitate the assistance of trained healthcare staff. As the majority of patients presented with palpitations or chest pain, mobile ECGs might contribute to improving care in these patient populations. In this study, the majority of patients with palpitations had a change in medical therapy. Innovations in the delivery of medication, for example the pill-in-the-pocket, might facilitate initial treatment at home. As such, the use of e-Health for remote diagnosis is worth investigating.

\section{Limitations}

This study was limited by the fact that data collection was done in a single tertiary medical centre, which could potentially affect generalisability. No data from other hospitals were incorporated in this study. Therefore, data on healthcare utilisation presented in this study might be an underestimation, as GUCH patients that participated could have been admitted to other hospitals. Lastly, 16 patients in our study had a high emergency care utilisation. This sample size is relatively small and the percentages derived from this sample should therefore be interpreted with caution.

\section{Planned healthcare utilisation}

This study was primarily concerned with the role of mHealth to decrease emergency care utilisation. It might, however, be possible that frequent collection of vital signs and remote doctor-patient contact will decrease the need for planned in-office visits as well. Moreover, mHealth could also contribute to the improvement of patient satisfaction and patient health engagement [23]. This should be measured in future mHealth initiatives as well.

\section{Conclusion}

GUCH patients who are symptomatic, those on antiarrhythmic drug therapy and those on diuretics are optimal candidates for enrolment in new mHealth initiatives because of both a high care utilisation and high motivation. Our study contributes to appropriate patient selection for mHealth initiatives that aim to prevent emergency care utilisation, thereby contributing to an efficient use of mHealth.

Acknowledgements The work described in this study was carried out in the framework of both the e-Health Citrien program and the Parelsnoer Institute (PSI). The e-Health Citrien program and PSI are part of and funded by the Dutch Federation of University Medical Centres (NFU).

Conflict of interest R.W. Treskes, M. Koole, D. Kauw, M.M. Winter, M. Monteiro, D. Dohmen, A. Abu-Hanna, M.P. Schijven, B.J. Mulder, B.J. Bouma and M.J. Schuuring declare that they have no competing interests.

Ethical approval For the collection of medical data from all the participating GUCH patients, permission was granted by the Ethics Committee (reference number W16_057). For the questionnaire survey no approval from the Institutions' Ethics Committee was required under Dutch law, since it was not burdensome for the patients.

Open Access This article is distributed under the terms of the Creative Commons Attribution 4.0 International License (http://creativecommons.org/licenses/by/4.0/), which permits unrestricted use, distribution, and reproduction in any medium, provided you give appropriate credit to the original author(s) and the source, provide a link to the Creative Commons license, and indicate if changes were made.

\section{References}

1. TennantPW,PearceMS, BythellM, RankinJ.20-yearsurvival of children born with congenital anomalies: a populationbased study. Lancet. 2010;375(9715):649-56.

2. Canfield MA, Honein MA, YuskivN, etal. National estimates and race/ethnic-specific variation of selected birth defects 
in the United States, 1999-2001. Birth Defects Res Part A Clin Mol Teratol. 2006;76(11):747-56.

3. Bird TM, Hobbs CA, Cleves MA, Tilford JM, Robbins JM. National rates of birth defects among hospitalized newborns. BirthDefects Res PartAClin Mol Teratol. 2006;76(11):762-9.

4. Baumgartner H, Bonhoeffer P, De Groot NM, et al. ESC guidelines for the management of grown-up congenital heart disease (new version 2010). Eur Heart J. 2010;31(23):2915-57.

5. Koyak Z, Kroon B, de Groot JR, et al. Efficacy of antiarrhythmic drugs in adults with congenital heart disease and supraventricular tachycardias. Am J Cardiol. 2013;112(9):1461-7.

6. Schuuring MJ, van Gulik EC, Koolbergen DR, et al. Determinants of clinical right ventricular failure after congenital heart surgery in adults. J Cardiothorac Vasc Anesth. 2013;27(4):723-7.

7. Zomer AC, Vaartjes I, van der Velde ET, et al. Heart failure admissions in adults with congenital heart disease; risk factors and prognosis. Int JCardiol. 2013;168(3):2487-93.

8. Schuuring MJ, van Riel AC, Vis JC, et al. New predictors of mortality in adults with congenital heart disease and pulmonary hypertension: midterm outcome of a prospective study. Int J Cardiol. 2015;181:270-6.

9. Tutarel O, Kempny A, Alonso-Gonzalez R, et al. Congenital heart disease beyond the age of 60: emergence of a new population with high resource utilization, high morbidity, and high mortality. Eur HeartJ. 2014;35(11):725-32.

10. Cowie MR, Bax J, Bruining N, et al. e-Health: a position statement of the European Society of Cardiology. Eur Heart J. 2016;37(1):63-6.

11. Treskes RW, van der Velde ET, Barendse R, Bruining N. Mobile health in cardiology: a review of currently available medical apps and equipment for remote monitoring. Expert Rev Med Devices. 2016;13(9):823-30.

12. Dorsey ER, Topol EJ. State of telehealth. N Engl J Med. 2016;375(2):154-61.
13. Clark PA, Capuzzi K, Harrison J. Telemedicine: medical, legal and ethical perspectives. Med Sci Monit. 2010;16(12):RA261-RA72.

14. Warnes CA, Liberthson R, Danielson GK, et al. Task force 1: the changing profile of congenital heart disease in adult life. JAm Coll Cardiol. 2001;37(5):1170-5.

15. Schuuring MJ, Backx AP, Zwart R, et al. Mobile health in adults with congenital heart disease: current use and future needs. Neth Heart J. 2016;24(11):647-52.

16. Mackie AS, Pilote L, Ionescu-Ittu R, Rahme E, Marelli AJ. Health care resource utilization in adults with congenital heart disease. Am J Cardiol. 2007;99(6):839-43.

17. Verheugt CL, Uiterwaal CS, van der Velde ET, et al. The emerging burden of hospital admissions of adults with congenital heart disease. Heart. 2010;96(11):872-8.

18. Cedars AM, Burns S, Novak EL, Amin AP. Predictors of rehospitalization among adults with congenital heart disease are lesion specific. Circ Cardiovasc Qual Outcomes. 2016;9(5):566-75.

19. Negishi J, Ohuchi H, Yasuda K, Miyazaki A, Norifumi N, Yamada O. Unscheduled hospitalization in adults with congenital heart disease. Korean Circ J.2015;45(1):59-66.

20. Moons P, Siebens K, De Geest S, Abraham I, Budts W, Gewillig M. A pilot study of expenditures on, and utilization of resources in, health care in adults with congenital heart disease. CardiolYoung. 2001;11(3):301-13.

21. Al-Zaiti SS, Shusterman V, Carey MG. Novel technical solutions for wireless ECG transmission \& analysis in the age of the internet cloud. JElectrocardiol. 2013;46(6):540-5.

22. Gordon NP, Hornbrook MC. Differences in access to and preferences for using patient portals and other ehealth technologies based on race, ethnicity, and age: a database and survey study of seniors in a large health plan. J Med Internet Res. 2016;18(3):e50.

23. De Rosis S, Barsanti S. Patient satisfaction, e-health and the evolution of the patient-general practitioner relationship: evidence from an Italian survey. Health Policy (New York). 2016;120(11):1279-92. 


\title{
Advertisement placed here.
}

\author{
ces bohn \\ CL van loghum
}

Houten 2019 


\title{
Advertisement placed here.
}

\author{
ces bohn \\ CL van loghum
}

Houten 2019 